\begin{tabular}{|c|l|}
\hline Title & $\begin{array}{l}\text { Emergence of a linear slope region of the isotherm in the first-order liquid-expanded-liquid-condensed phase transition } \\
\text { in Langmuir monolayers }\end{array}$ \\
\hline Author(s) & Hatta, Eiji; Nihei, Ko \\
\hline Citation & $\begin{array}{l}\text { Physical Review E, 100(2), 022801 } \\
\text { https://doi.org/10.1103/PhysRevE.100.022801 }\end{array}$ \\
\hline Issue Date & 2019-08-01 \\
\hline Doc URL & http://hdl.handle.net/2115/75367 \\
\hline Rights & O2019A A merican Physical Society \\
\hline Type & article \\
\hline File Information & PhysRevE.100.022801.pdf \\
\hline
\end{tabular}

Instructions for use 


\title{
Emergence of a linear slope region of the isotherm in the first-order liquid-expanded-liquid-condensed phase transition in Langmuir monolayers
}

\author{
Eiji Hatta $\oplus^{*}$ and Ko Nihei \\ Nanoelectronics Laboratory, Graduate School of Information Science and Technology, Hokkaido University, Sapporo, 060-0814, Japan
}

(Received 23 May 2019; revised manuscript received 14 July 2019; published 1 August 2019)

\begin{abstract}
A nonhorizontal slope in the isotherm has been observed in the two-phase coexisting region of the firstorder liquid-expanded (LE)-liquid-condensed (LC) phase transition in Langmuir monolayers for many decades. We show that the simple analysis of a phenomenological Landau free energy involving the coupling-energy contributions of molecular lateral density $(\rho)$ with spontaneous collective chain tilt $(\theta)$ and two-dimensional strain $\left(\varepsilon_{s}\right)$ inside the LC domain enables one to understand the origin of a nonhorizontal straight-line slope in the LE-LC phase coexistence region of the isotherm. The presence of $\rho-\varepsilon_{s}$ coupling must be essential for the appearance of the straight-line shape of a nonhorizontal plateau in the isotherm. Moreover, it is found from the comparison of the two-dimensional contour plots of the free energy that an LE phase may persist significantly even at the later stage of the straight-line regime beyond a transition midpoint surface pressure in the presence of this coupling. The persistence of the LE phase may lead to the delay of transition progress as manifested more clearly by the appearance of a compressibility plateau in the coexistence region that indicates the existence of persistent equilibrium density fluctuations in the monolayer.
\end{abstract}

DOI: 10.1103/PhysRevE.100.022801

\section{INTRODUCTION}

Langmuir monolayers have received much attention from the viewpoints of physical, chemical, biological interests, and their potential applications [1-4]. Surface pressure $(\pi)$ -molecular area $(A)$ isotherms provide information about the phases and phase transitions in monolayers [5]. The isotherms, characterized by the appearance of a nonhorizontal slope region followed by a steeper nonlinear ascent region in the phase-coexistence region of a first-order liquid-expanded (LE)-liquid-condensed (LC) phase transition, have been most commonly found in some fatty-acid monolayers [6] and in phospholipid monolayers $[7,8]$. The origin of a nonzero slope in the isotherm has been extensively discussed from various perspectives: (i) the effect of impurities in monolayer materials $[9,10]$, (ii) the effect of the long-range dipole interaction $[11,12]$ and (iii) the formation of two-dimensional molecular aggregates such as surface micelles and submicroscopic clusters constituting a finite number of amphiphilic molecules [13-15]. Besides the above possibilities, some authors discussed the nonhorizontal slope of isotherms from certain mechanical viewpoints. Arriaga et al. stressed the significance of the mechanical rigidity of a microheterogeneous composite medium composed of two coexisting phases to the occurrence of a finite slope in the phase-coexisting region [16].

As mentioned above, although the origin of the appearance of a nonhorizontal slope in the isotherm has been extensively discussed from both theoretical and experimental viewpoints (and it still remains unclear), another interesting puzzle to be solved in a monolayer isotherm concerns the shape of a nonhorizontal slope, which has almost not been addressed

*hatta@ist.hokudai.ac.jp so far. In this paper we focus our attention on the origin of the isotherm shape of a nonhorizontal slope appearing immediately after the onset of the LE to LC phase transition. The isotherm generally exhibits either one of two types of shapes in the nonhorizontal slope region: a curved line or a straight line. The isothermal compressibility, defined as the fractional change of surface area per unit change in surface pressure, characterizes the isotherm shape in the LE-LC phase-coexistence region more definitely. It normally shows a peak $[17,18]$ or a plateau $[13,19,20]$ corresponding to a curved line or a straight line in the isotherm, respectively (Fig. 1). The cause of a maximum in the experimental compressibility curve in an LE-LC transition was discussed in relation to a change in the tilt of the hydrocarbon chain at the air-water interface and to reorientations in the head group region [17,21]. From a theoretical point of view, the formation of a finite size of cooperatively transforming molecular clusters as small systems was attributed to a phase transition over some range of pressures and this transition would be possible to exhibit a finite slope in the isotherm [14,22]. In this case, however, the resultant compressibility curves always must show a peak and not a plateau, as it can be easily checked by direct calculation from the cooperative cluster model [14,22].

We must thus seek another cause for the origin of the occurrence of a nonhorizontal straight-line shape in the isotherm and of the resultant compressibility plateau. We here note that the ordered fluid LE-LC phase transition is a kind of first-order transition between condensed phases and that the transition proceeds by the growth of domains of the product phase at the expense of the surrounding parent phase [16]. In monolayer isotherms in which the LE-LC phase-coexistence region can be observed, the area of a molecule $\left(A_{\mathrm{LE}}\right)$ in the LE phase is normally much greater than that $\left(A_{\mathrm{LC}}\right)$ in the LC phase. In the phase-coexistence region it is thus highly 


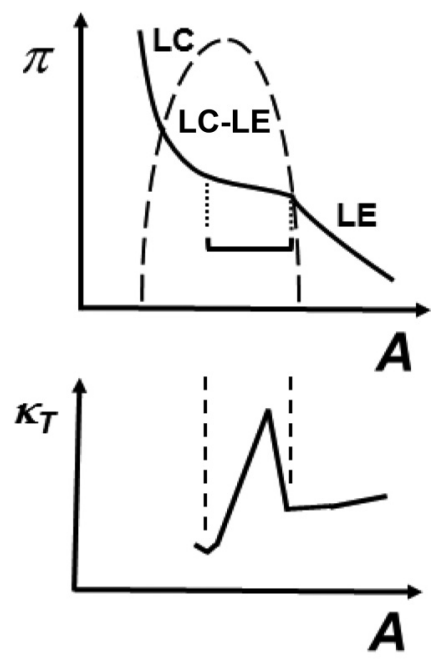

or

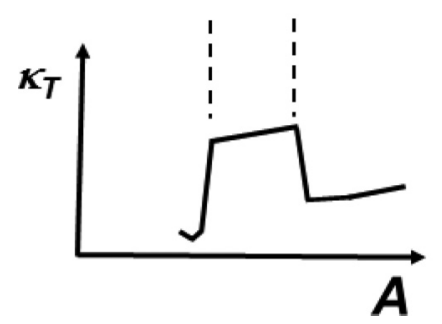

FIG. 1. Schematic illustration of a monolayer isotherm and two types of compressibilities in the two-phase coexistence region of the first-order LE-LC phase transition in Langmuir monolayers.

likely that the free energy of an LC domain within a matrix of LE phase is significantly different from that of an LE domain of exactly the same size and shape within a matrix of LC phase due to its different stored strain energy. Therefore an additional mechanical strain energy contribution must be involved in the excess free-energy expression of the LE-LC transition. In Ref. [16] Arriaga et al. indeed investigated the macroscopic relation between the mechanical rigidity kinetically controlled of a microheterogeneous medium constituted of two coexisting phases and frustrated first-order metastable energetics giving rise to a nonhorizontal transition plateau in the isotherm. Kaganer et al. reported experimental evidence of an equilibrium phase coexistence in strained heteroepitaxial films over a wide temperature interval [23,24]. The fraction of the low-temperature phase decreased almost linearly with approaching the phase-transition temperature. We expect that such a mechanical strain-related first-order phase transition may occur over some temperature or pressure range in the LE-LC transition.

From the above viewpoints, in this study we present a simple argument based on a phenomenological ("coarse- grained") Landau free energy involving the coupling effects of molecular lateral density $(\rho)$ with spontaneous molecular collective chain tilt $(\theta)$ and two-dimensional (2D) strain $\left(\varepsilon_{s}\right)$ inside the LC domain to examine the effects of stored strain energy on the first-order LE-LC phase transition in Langmuir monolayers. As the result of this analysis, it is found that the existence of $\rho-\varepsilon_{s}$ coupling in the transition region may lead to the appearance of a nonhorizontal linear slope region in the isotherm with equilibrium phase fractions depending on the surface pressure. The comparison of the $2 \mathrm{D}$-contour plots of the free energy calculated with different renormalization parameter values suggests that $\rho-\varepsilon_{s}$ coupling leads to the persistence of the LE phase even at the later stage of the linear slope regime beyond a transition midpoint surface pressure in the two-phase coexistence region. This may be closely related to the appearance of an isothermal compressibility plateau as often previously observed in lipid monolayers [19,20,25].

\section{LANDAU MODEL INVOLVING THE COUPLING-ENERGY TERMS OF LATERAL DENSITY WITH SPONTANEOUS CHAIN TILT AND 2D STRAIN FOR A FIRST-ORDER LE-LC PHASE TRANSITION IN LANGMUIR MONOLAYERS}

\section{A. Formulation}

The magnitudes of spontaneous molecular collective chain tilt $(\theta)$ and 2D strain $\left(\varepsilon_{S}\right)$ inside the LC domain in monolayer can depend on the extent of an LE to LC transition progress, and the presence of $\theta$ and $\varepsilon_{s}$, on the other hand, can affect the degree to which the transition proceeds. There must thus be a corresponding decrease in the free energy through the evolution of $\rho$ in such a way as to compensate the excess elastic energy for $\theta$ and $\varepsilon_{s}$ generated within the monolayer. That is, the changes of $\rho, \theta$, and $\varepsilon_{s}$ must be adjusted spontaneously to minimize the total free-energy penalty as the transition proceeds. In order to get direct and intuitive insights into the macroscopic thermodynamic character of such a firstorder LE-LC phase transition under the growth of internal strain and the microscopic driving forces behind it, let us start with a phenomenological Landau model [26]. Since the generation of a long-range strain field due to $\rho-\varepsilon_{s}$ coupling promotes the mean-field behavior, a Landau theory is expected to provide an accurate description of phase transitions in a monolayer under influence of strain.

In order to investigate the manner in which spontaneous orientational elasticity and 2D strain fields influence the progress of the first-order transition in monolayer with some pseudo-2D character, we assume that the excess free energy of the low-symmetry LC phase over that of the high-symmetry LE phase is given as follows:

$$
\begin{aligned}
\Delta G(\pi, T)= & G_{\mathrm{LC}}-G_{\mathrm{LE}}=\frac{1}{2} a\left(T-T_{0}\right) \rho^{2}+\frac{1}{4} B \rho^{4}+\frac{1}{6} C \rho^{6} \quad(a, C>0, \quad B<0) \\
& +\frac{1}{2} K_{\theta} \theta^{2}+\frac{K_{0} \varepsilon_{\mathrm{s}}^{2}}{2} \frac{1+\gamma_{0}}{1-v} \quad v=\left(\frac{R_{\mathrm{LC}}}{R_{\mathrm{LE}}}\right)^{2}, \quad \gamma_{0}=\frac{4}{3} \frac{\mu_{0}}{K_{0}}, \quad\left(K_{0}, K_{\theta}>0, \varepsilon_{\mathrm{s}}, \mu_{0} \geqslant 0\right) \quad\left(\Delta G_{\rho}\right) \\
& +d \rho^{2} \theta+e \rho^{2} \varepsilon_{\mathrm{s}} \quad(d, e \geqslant 0)
\end{aligned}
$$


In Eq. (1) the order parameter driving the transition in monolayer is the lateral packing density difference $\rho(=$ $\left.\rho_{\mathrm{LC}}-\rho_{\mathrm{LE}}\right)$ between the two phases in order to account for a large change in molecular lateral density (generally one order of magnitude higher than density differences between the liquid and solid phases of bulk materials). A possible LE-LC phase boundary (line energy) effect can be neglected in the present case since it is small compared to the long-ranged strain energy. $\Delta G_{\rho}$ is a standard Landau potential for the variation of excess free energy with temperature. This has only even powers from symmetry reasons. $a, B$, and $C$ are coefficients which do not depend explicitly on temperature and surface pressure. We choose $B<0$ and $C>0$ to consider the effects of chain tilt and 2D strain on the molecular density in monolayer for the case of an already first-order transition. We note that $T_{0}$ is the equilibrium transition temperature for second-order and tricritical transitions under ordinary pressure and that a first-order transition occurs at a higher transition temperature $T_{\mathrm{tr}}\left(=T_{0}+(3 / 16)\left(B^{2} / a C\right)>T_{0}\right)$.

In addition to $\Delta G_{\rho}$, we have added excess chain tilt-2D strain energy $\Delta G_{\text {elastic }}$ and excess molecular density-chain tilt-2D strain coupling energy $\Delta G_{\text {coupling }}$ in the total excess free energy $\Delta G . K_{0}$ and $\mu_{0}$ are the compression and shear moduli of the LE phase and $\varepsilon_{\mathrm{s}}$ is the $2 \mathrm{D}$ strain inside the LC phase. $K_{\theta}$ is the orientation (splay) elastic constant. Although $\mu_{0}$ is normally negligibly small compared to $K_{0}$ in the LE phase [16,27], we retain $\gamma_{0}\left(=(4 / 3)\left(\mu_{0} / K_{0}\right)\right)$ term for generality. $v$ is the area fraction of the LC phase to the total area, $v=S_{\mathrm{LC}} /\left(S_{\mathrm{LE}}+S_{\mathrm{LC}}\right)=\left(R_{\mathrm{LC}} / R_{\mathrm{LE}}\right)^{2}$ (see Fig. 6 in Appendix A). Fluorescence microscopy image analysis combined with the "lever rule" technique demonstrated that $v$ is inversely proportional to $A$ in the LE-LC phase coexistence region $[28,29]$. In the present model, one can thus regard $v$ as a direct counterpart to the average molecular density $\left(A^{-1}\right)$ in the isotherm. That is, in the phase-coexistence region, $v=0$ corresponds to the molecular area $A_{\mathrm{LE}}$ for a single LE phase, i.e., the onset surface area of the LE to LC transition and $v \rightarrow 1$ means that the molecular area approaches the molecular area $A_{\mathrm{LC}}$ for a single LC phase. $\Delta G_{\text {elastic }}$ in Eq. (1) involves spontaneous strain-energy terms derived from the orientation and the 2D Hooke's laws, assuming that their changes are small through the transition. The first term in $\Delta G_{\text {elastic }}$ represents the excess elastic energy caused by the orientational change of molecules. In the collective change in equilibrium tilt angle, $\theta=\theta_{\mathrm{LC}}-\theta_{\mathrm{LE}}$, resulting from intermolecular interactions between the tail groups, $\theta_{\mathrm{LE}}$ and $\theta_{\mathrm{LC}}$ are the angles between the average chain orientation and the normal to the water surface in each phase. The second term in $\Delta G_{\text {elastic }}$ is the excess 2D strain energy of the LC phase depending on the LC phase area ratio $v$, and it was derived within the linear elasticity framework under the assumption that the monolayer is under hydrostatic compression (see Appendix A for the derivation). The "renormalized" compression modulus, $K^{\prime}=\frac{1+\gamma_{0}}{1-\nu} K_{0}$, indicates that elastic hardening of the two-phase coexistence system gradually develops as the LE to LC transition proceeds. The value of $\rho$ will be influenced by $\theta$ and $\varepsilon_{s}$ during the transition. $\Delta G_{\text {coupling in Eq. (1) represents }}$ the coupling contributions of $\theta$ and $\varepsilon_{s}$ to $\rho$. The geometrical parameters, $\theta$ and $\varepsilon_{s}$, are allowed to be coupled with $\rho$ in the lowest order by symmetry reasons. The coupling constants $d$ and $e$ express the strength of coupling of $\rho$ with $\theta$ and $\varepsilon_{s}$, respectively. We have ignored the complexities introduced by $\pi, T$ dependences of the coupling constants $d, e$ and of the elastic moduli $K_{0}, K_{\theta}$ for simplicity. The first terms in $\Delta G_{\text {elastic }}$ and $\Delta G_{\text {coupling }}$ were first introduced by Albrecht and coauthors in order to investigate the chain-melting transition in phospholipid monolayers [13].

The Landau expansion is normally described for phase transitions that occur as temperature is varied under ordinary pressure. It would be convenient to convert the external intensive variable from temperature to surface pressure in $\Delta G_{\rho}$ in order to consider a monolayer isotherm. After some manipulation of Eq. (1) (Appendix B), we finally obtain the rescaled free energy, $\Delta G_{v}^{\text {res: }}$

$$
\begin{aligned}
\Delta G_{v}^{\mathrm{res}} & =\left(\frac{C^{2}}{|B|^{3}}\right) \Delta G_{v} \\
& =v\left(\frac{\pi^{\mathrm{res}}}{2} \xi^{2}-\frac{1}{4}\left(1+\alpha+\beta \frac{1-v}{1+\gamma_{0}}\right) \xi^{4}+\frac{1}{6} \xi^{6}\right), \\
\rho & =\left(\frac{|B|}{C}\right)^{1 / 2} \xi, \quad \pi^{\mathrm{res}}=-\frac{a C}{B^{2}}\left(\frac{d T_{\mathrm{c}}}{d \pi_{\mathrm{c}}}\right)\left(\pi-\pi_{\mathrm{c}}\right), \\
\alpha & =\frac{2 d^{2}}{|B| K_{\vartheta}} \geqslant 0, \quad \beta=\frac{2 e^{2}}{|B| K_{0}} \geqslant 0, \quad \gamma_{0}=\frac{4 \mu_{0}}{3 K_{0}} \geqslant 0 .
\end{aligned}
$$

Here we note that the actual surface pressure $\pi\left(<\pi_{\mathrm{c}}\right)$ increases as the rescaled surface pressure $\pi^{\text {res }}$ decreases since the coefficient $-\left(a C / B^{2}\right)\left(d T_{\mathrm{c}} / d \pi_{\mathrm{c}}\right)$ is always negative under the condition considered here.

\section{B. Analysis of the rescaled Landau free energy}

Using the rescaled free energy, Eq. (2) and from the condition $\partial \Delta G_{v}^{\text {res }} / \partial \xi=0, \pi^{\text {res }}$ can be expressed by the rescaled order parameter $\xi$ as follows:

$$
\begin{aligned}
& \frac{\partial \Delta G_{\nu}^{\mathrm{res}}}{\partial \xi}=\nu \xi\left\{\pi^{\mathrm{res}}-\left(1+\alpha+\beta \frac{1-v}{1+\gamma_{0}}\right) \xi^{2}+\xi^{4}\right\}=0 \\
& \therefore \pi^{\mathrm{res}}=\left(1+\alpha+\beta \frac{1-v}{1+\gamma_{0}}\right) \xi^{2}-\xi^{4} .
\end{aligned}
$$

Substituting $\pi^{\text {res }}$ in Eq. (3) into that in Eq. (2) and from the condition $\partial \Delta G_{v}^{\text {res }} / \partial v=0$, one can obtain the equilibrium order parameters $\xi_{\mathrm{LE}}$ and $\xi_{\mathrm{LC}}$ :

$$
\begin{aligned}
& \frac{\partial \Delta G_{v}^{\mathrm{res}}}{\partial v}=\xi^{4}\left\{-\frac{1}{3} \xi^{2}+\frac{1}{4}(1+\alpha)+\frac{\beta}{4}\left(\frac{1-2 v}{1+\gamma_{0}}\right)\right\}=0 . \\
& \therefore \xi_{\mathrm{LE}}=0 \quad \text { and } \quad \xi_{\mathrm{LC}}= \pm\left\{\frac{3}{4}\left(1+\alpha+\beta \frac{1-2 v}{1+\gamma_{0}}\right)\right\}^{1 / 2} .
\end{aligned}
$$

Since the order parameter $\xi_{\mathrm{LC}}$ must be real and nonzero in the phase coexistence, we impose the condition of the radicand in Eq. (4) being real and positive in the phasecoexistence region between $v=0.0$ and $v=1.0$. The range of $\beta$ is hence bounded above by the condition $1+\alpha>\beta$. At the transition midpoint, the condition $\Delta G_{v}^{\text {res }}=0$ should be satisfied. Using the nonzero $\xi_{\mathrm{LC}}$ in Eq. (4), we have $v$ 
dependence on $\pi^{\text {res: }}$

$$
\begin{aligned}
& \nu \xi^{2}\left\{\frac{\pi^{\mathrm{res}}}{2}-\frac{1}{4}\left(1+\alpha+\beta \frac{1-v}{1+\gamma_{0}}\right) \xi^{2}+\frac{1}{6} \xi^{4}\right\}=0, \\
& \therefore \pi_{\nu}^{\mathrm{res}}=\frac{1}{2}\left(1+\alpha+\beta \frac{1-v}{1+\gamma_{0}}\right) \xi^{2}-\frac{1}{3} \xi^{4} \\
& =\frac{3}{16}\left(1+\alpha+\beta \frac{1}{1+\gamma_{0}}\right)\left(1+\alpha+\beta \frac{1-2 v}{1+\gamma_{0}}\right) .
\end{aligned}
$$

From $\pi_{\nu}^{\text {res }}$ in Eq. (5) and the relation between $\pi^{\text {res }}$ and $\pi$ in Eq. (2), we can obtain the following important result: In the presence of molecular density-2D strain coupling $(\beta \neq 0)$, the actual surface pressure $\pi$ increases "linearly" with increasing (decreasing) molecular density (molecular area) in a nonhorizontal plateau of the two-phase coexistence region of the isotherm.

From Eqs (2), (4), and (5), we obtain the following scaling relation between $\xi_{\mathrm{LC}}$ and $\pi$ :

$$
\begin{aligned}
\xi_{\mathrm{LC}}= & \pm 2\left(\frac{a C}{B^{2}}\right)^{1 / 2}\left(\frac{d T_{\mathrm{c}}}{d \pi_{\mathrm{c}}}\right)^{1 / 2}\left(1+\alpha+\beta \frac{1}{1+\gamma_{0}}\right)^{-1 / 2} \\
& \times\left(\pi_{\mathrm{c}}-\pi\right)^{1 / 2} .
\end{aligned}
$$

Using $\pi_{v}^{\text {res }}$ in Eq. (5), the onset surface pressure of the LE to $\mathrm{LC}$ transition $(v=0)$,

$$
\begin{aligned}
\pi_{\nu=0}^{\mathrm{res}} & =\frac{1}{2}\left(1+\alpha+\beta \frac{1}{1+\gamma_{0}}\right) \xi^{2}-\frac{1}{3} \xi^{4} \\
& =\frac{3}{16}\left(1+\alpha+\beta \frac{1}{1+\gamma_{0}}\right)^{2} .
\end{aligned}
$$

At the transition midpoint $(v=0.5)$,

$$
\begin{aligned}
\pi_{\mathrm{tr}}^{\mathrm{res}} \equiv \pi_{\nu=0.5}^{\mathrm{res}} & =\frac{1}{2}+(1+\alpha) \xi^{2}-\frac{1}{3} \xi^{4} \\
& =\frac{3}{16}\left(1+\alpha+\beta \frac{1}{1+\gamma_{0}}\right)(1+\alpha) .
\end{aligned}
$$

Therefore, the actual transition midpoint surface pressure $\pi_{\mathrm{tr}}$ is

$$
\begin{aligned}
\pi_{\mathrm{tr}} & =\pi_{\mathrm{c}}-\pi_{\mathrm{tr}}^{\mathrm{res}}\left(\frac{B^{2}}{a C}\right)\left(\frac{d \pi_{\mathrm{c}}}{d T_{\mathrm{c}}}\right) \\
& =\pi_{\mathrm{c}}-\frac{3}{16} \frac{B^{2}}{a C}\left(\frac{d \pi_{\mathrm{c}}}{d T_{\mathrm{c}}}\right)\left(1+\alpha+\beta \frac{1}{1+\gamma_{0}}\right)(1+\alpha) .
\end{aligned}
$$

From Eq. (9) the strain-renormalized, actual surface pressure $\pi_{\text {tr }}$ becomes lower compared to the corresponding "bare" surface pressure $\pi_{\mathrm{tr}}^{0}\left(=\pi_{\mathrm{c}}-(3 / 16)\left(B^{2} / a C\right)\left(d \pi_{\mathrm{c}} / d T_{\mathrm{c}}\right)\right)$ for $\alpha \neq 0$ and/or $\beta \neq 0$.

At the later stage of the straight-line regime of the transition (for instance, at $v=0.8$ ):

$$
\begin{aligned}
\pi_{\nu=0.8}^{\mathrm{res}} & =\frac{1}{2}(1+\alpha) \xi^{2}-\frac{1}{3} \xi^{4} \\
& =\frac{3}{16}\left(1+\alpha+\beta \frac{1}{1+\gamma_{0}}\right)\left(1+\alpha-0.6 \beta \frac{1}{1+\gamma_{0}}\right) .
\end{aligned}
$$
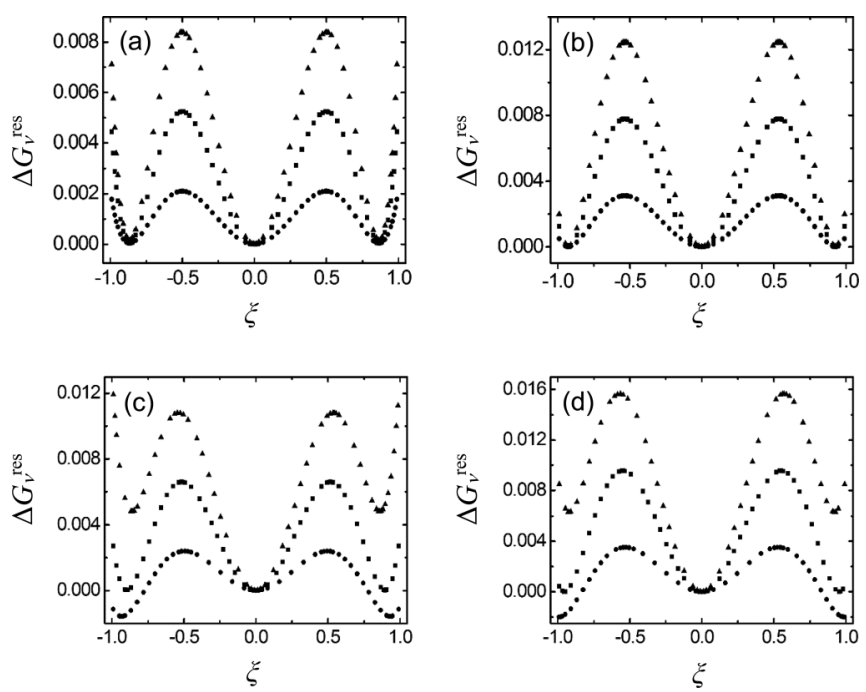

FIG. 2. 2D-contour plots of the Landau free energy $\Delta G_{v}^{\text {res }}$ [Eq. (2)] along the rescaled lateral density order parameter $\xi$ and the LC phase area ratios $v$ : (a) $\alpha=0.0 ; \beta=0.0, \gamma_{0}=0.0, \pi_{\mathrm{tr}}^{\text {res }}=$ 0.188 (decoupling); (b) $\alpha=0.143, \beta=0.0, \gamma_{0}=0.0, p_{\text {tr }}{ }^{\text {res }}=0.245$ (density-chain tilt coupling); (c) $\alpha=0.0, \beta=0.143, \gamma_{0}=0.0$, $\pi_{\mathrm{tr}}^{\text {res }}=0.214$ (density-2D strain coupling); (d) $\alpha=0.143, \beta=$ $0.143, \gamma_{0}=0.0, \pi_{\mathrm{tr}}{ }^{\text {res }}=0.276$ (density-chain tilt-2D strain coupling). (๑) $v=0.2 ;(\mathbf{\square}) v=0.5 ;(\mathbf{\Delta}) v=0.8$.

From Eqs. (2), (7), and (10), as the transition proceeds from $v=0.0$ to $v=0.8$, the actual surface pressure interval $\Delta \pi$ is

$$
\begin{aligned}
\Delta \pi & =\pi_{\nu=0.8}-\pi_{\nu=0} \\
& =-\frac{B^{2}}{a C}\left(\frac{d \pi_{\mathrm{c}}}{d T_{\mathrm{c}}}\right)\left(\pi_{\nu=0.8}^{\mathrm{res}}-\pi_{\nu=0}^{\mathrm{res}}\right) \\
& =\frac{3}{10} \frac{B^{2}}{a C}\left(\frac{d \pi_{\mathrm{c}}}{d T_{\mathrm{c}}}\right) \frac{\beta}{\left(1+\gamma_{0}\right)^{2}}\left\{(1+\alpha)\left(1+\gamma_{0}\right)+\beta\right\} .
\end{aligned}
$$

For the decoupling limit $(\beta \rightarrow 0)$ between $\rho$ and $\varepsilon_{\mathrm{s}}, \Delta \pi$ approaches zero; that is, a nonhorizontal slope of $2 \mathrm{D}$ strain origin does vanish in the isotherm.

\section{RESULTS}

To investigate the geometrical features of our derived free energy $\Delta G_{v}^{\text {res }}$ [Eq. (2)], we show the two-dimensional contour plots of the free energy along the rescaled lateral density order parameter $\xi$ and for some typical LC phase area ratios $v$ in the two-phase coexistence region (Fig. 2). $\Delta G_{v}^{\text {res }}$ is an even function of $\xi$ and it is symmetric with respect to $\xi=0.0$. Each 2D free-energy contour plot was made with the equilibrium transition surface pressure $\pi_{\mathrm{tr}}^{\mathrm{res}}$ [Eq. (8)] for given $\alpha, \beta, \gamma_{0}$ values. The values of $\alpha, \beta, \gamma_{0}$ were chosen arbitrarily for illustration of the effects of molecular density-mechanical strain coupling on $\Delta G_{v}^{\text {res }}$. The LE and the LC phases correspond to the free-energy minima at $\xi_{\mathrm{LE}}=0$ and at some nonzero $\xi_{\mathrm{LC}}$, respectively. They have the same energy value at $v=0.5$ for all cases. There is one free-energy maximum constituting a finite energy barrier between the two free-energy minima. The energy barrier height depends on the strength of both $\rho-\theta$ 

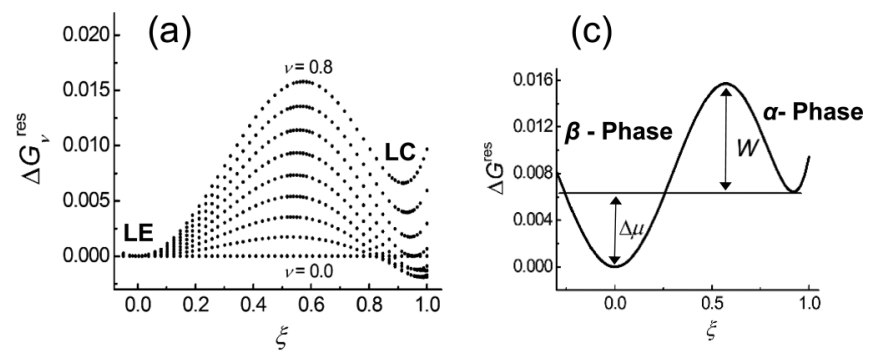

(b)

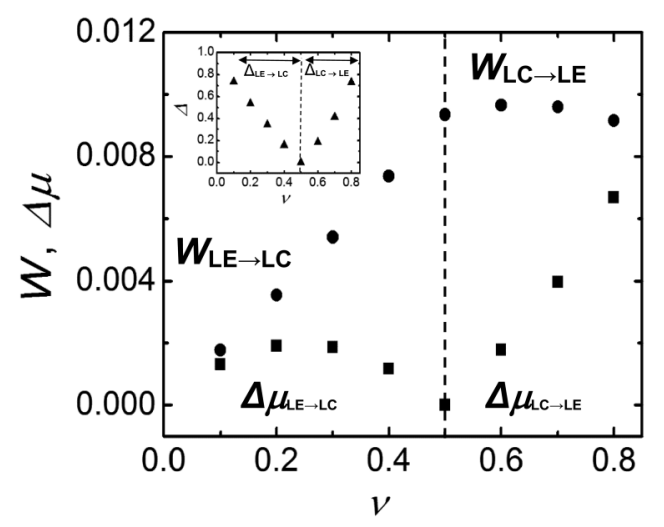

FIG. 3. (a) $2 \mathrm{D}$-contour plot $(\xi \geqslant 0.0)$ of the Landau free energy shown in $(d)$. The two minima correspond to the $\operatorname{LE~}(\xi=0)$ and the LC $(\xi \neq 0)$ phases, respectively. (b) The LC phase area ratio $v$ dependence on the energy barrier height $W(\bullet)$ and the energy asymmetry $\Delta \mu(\mathbf{\square})$. Inset: The LC phase area ratio $v$ dependence on the driving force $\Delta(\equiv \Delta \mu / W)(\mathbf{\Delta})$ for transition. (c) Schematic of the transition from a higher metastable $\alpha$ phase, over an energy barrier $W$ to a lower stable $\beta$ phase.

$(\alpha)$ and $\rho-\varepsilon_{\mathrm{S}}(\beta)$ couplings as well as on $\nu$. In the presence of $\rho-\varepsilon_{\mathrm{S}}$ coupling [Figs. 2(c) and 2(d)], the energy barrier for both $(\mathrm{LE} \rightarrow \mathrm{LC}$ and $\mathrm{LC} \rightarrow \mathrm{LE})$ directions is not identical except at $v=0.5$. We can see that the barrier asymmetry is reversed across at the transition midpoint $(v=0.5)$ and that the LC phase becomes energetically metastable above it. Figure 3(a) shows the 2D contour plot of the free energy shown in Fig. 2(d) at a smaller interval of $v$. In order to see this behavior more quantitatively we show $v$ dependence on the energy barrier height $W$ and the energy asymmetry $\Delta \mu$ [Fig. 3(b); see also Fig. 3(c)]. In the range of $v=0.0$ to $v=0.5$ the energy barrier height $W_{\mathrm{LE} \rightarrow \mathrm{LC}}$ from the majority LE phase to the minority LC phase increases monotonously with increasing $v$ while the energy asymmetry $\Delta \mu_{\mathrm{LE} \rightarrow \mathrm{LC}}$ does not change significantly. Above the midpoint, $W_{\mathrm{LC} \rightarrow \mathrm{LE}}$ from the majority LC phase to the minority LE phase remains almost constant while $\Delta \mu_{\mathrm{LC} \rightarrow \mathrm{LE}}$ increases monotonously. The driving force $\Delta$ for transition is plotted as a function of $v$ in the inset of Fig. 3(b). As the LC phase grows beyond the transition midpoint $(v>0.5)$, the driving force from the majority LC to the minority LE phase increases monotonously. In Fig. 4, we plot $v$ dependence on $\pi_{v}^{\text {res }}$ from Eq. (5) (remember that $v$ is a direct counterpart to the average molecular density, $A^{-1}$ ). In this plot we consider only the effect of densitymechanical (chain tilt-2D) strain coupling on the surface pressure. Although the effect of limited cooperativity in the

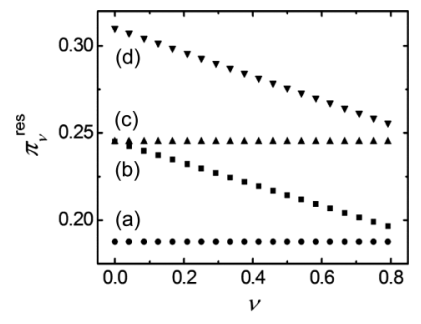

FIG. 4. LC phase area ratio $v$ dependence on rescaled equilibrium surface pressure $\pi_{v}^{\text {res }}$ : (a) $\alpha=0.0, \beta=0.0, \gamma_{0}=0.0$ (decoupling); (b) $\alpha=0.0, \beta=0.143, \gamma_{0}=0.0$ (density-2D strain coupling); (c) $\alpha=0.143, \beta=0.0, \gamma_{0}=0.0 \quad$ (density-chain tilt coupling); (d) $\alpha=0.143, \beta=0.143, \gamma_{0}=0.0$ (density-chain tilt-2D strain coupling).

transition would produce an additional contribution for the isotherm shape, its effect is not included in this plot. Only in the presence of $\rho-\varepsilon_{\mathrm{s}}$ coupling [Figs. 4(b) and 4(d)], $\pi_{v}^{\text {res }}$ decreases (or the actual surface pressure $\pi$ increases) linearly with increasing $v$ (or decreasing $A$ ) in the phase coexistence region. From Eq. (11), the stronger $\rho-\varepsilon_{\mathrm{s}}$ coupling (the larger $\beta$ ) is, the larger is the surface pressure interval $\Delta \pi$.

\section{DISCUSSION}

It is experimentally well known that cooperative clusters are formed in the LE-LC phase-coexistence region and that their size is normally finite (the order of 10-100 molecules) $[13,15,30,31]$. If this limited cooperativity is a dominant mechanism for the transition progress, the transition will certainly occur over some surface pressure range [14,22]. For such a finite cooperative transition, however, the isotherm must show a curved line shape in the coexistence region and the resultant compressibility curve must result in a peak with finite width and height. The presence of coupling of molecular lateral density $\rho$ with additional mechanical degrees of freedom, $\theta$ and $\varepsilon_{\mathrm{s}}$, might significantly affect its energetics in the LE-LC transition. In fact, if all the domains were to collect together to form one large domain, for instance, instead of a large number of separate small domains of one phase within the bulk, parent phase, then effects of strain energy would be remarkably reduced [32]. From the above considerations, we have investigated the energy cost of the formation of a finite fraction of LC region embedded inside an LE matrix within the framework of a phenomenological Landau model. The coupling effects of $\rho$ with spontaneous $\theta$ and $\varepsilon_{\mathrm{s}}$ can produce a strain renormalization of the fourth-order coefficient in the excess free energy $\Delta G_{v}^{\text {res }}$ [Eq. (2)]. This renormalization leads to the change in strength of the LE-LC transition and provides some useful information on how the LE to LC transition proceeds [Eq. (5)]. The fourth coefficient in $\Delta G_{v}^{\text {res }}$ increases negatively with increasing the renormalization constants $\alpha$ and $\beta$. This suggests that the transition is driven even more strongly first order with increasing the renormalization strength and that the actual transition pressure $\pi_{\mathrm{tr}}$ is reduced more strongly [Eq. (9)]. From Eqs. (2), (4), and (5), $v$ and the discontinuity in the order parameter $\Delta \xi\left(=\xi_{\mathrm{LC}}-\xi_{\mathrm{LE}}(\equiv 0)\right)$ scale with $\pi$ as $\nu \propto \pi$ and $\Delta \xi \propto\left(\pi_{\mathrm{c}}-\pi\right)^{1 / 2}$, respectively. The former relation is consistent with the previous experimental 


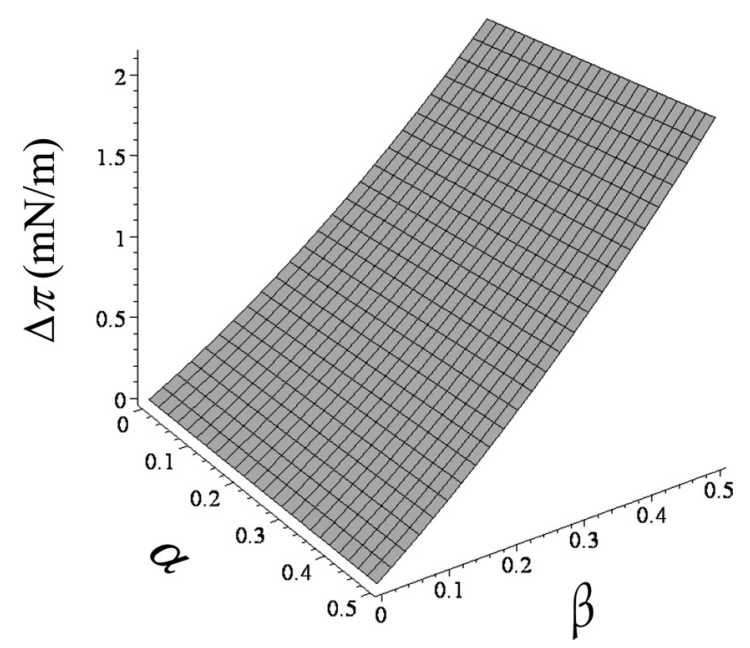

FIG. 5. Renormalized, control parameters $\alpha, \beta$ dependence on the equilibrium actual surface pressure difference $\Delta \pi$ in the twophase coexistence region of the LE-LC phase transition. $\left[B^{2} / a C=\right.$ $3.0 \mathrm{~K}, d \pi_{c} / d T_{c}=2.35 \mathrm{mNm}^{-1} \mathrm{~K}^{-1}, \gamma_{0}=0.0$ in Eq. (11)].

results $[13,19]$. From the latter scaling relation, as the LE to LC transition proceeds from $v=0$ to $v=0.8, \xi_{\mathrm{LC}}$ is expected to decrease by $1.6 \beta$ due to $\rho-\varepsilon_{\mathrm{s}}$ coupling. In the molecular density-mechanical strain decoupling limit $(\alpha, \beta \rightarrow 0)$, $\xi_{\mathrm{LC}}$ reduces to the bare order parameter $\rho_{\mathrm{LC}}^{0}=(3|B| / 4 C)^{1 / 2}$ at a single transition surface pressure $\pi_{\mathrm{tr}}^{0}\left(=\pi_{\nu=0}=\pi_{\nu=0.8}\right)=$ $\pi_{\mathrm{c}}-(3 / 16)\left(B^{2} / a C\right)\left(d \pi_{\mathrm{c}} / d T_{\mathrm{c}}\right)$. It should be noteworthy from Eqs. (5) and (11) that as far as a finite $\rho-\varepsilon_{\mathrm{s}}$ coupling $(\beta \neq 0)$ exists a finite isotherm linear slope must arise, irrelevant of the presence of density-chain tilt coupling. It was reported that the main (first-order) transition is characterized by the appearance of a nonhorizontal straight-line region in isotherms and isobars [13,19]. The appearance of a straightline shape of the isotherm in the LE-LC coexistence region is, however, nontrivial [20,33]. Our result indeed suggests that $\pi$ increases linearly with increasing $v$ (or with decreasing $A$ ) in the isotherm only in the presence of $\rho-\varepsilon_{\mathrm{s}}$ coupling. Although a nonhorizontal straight-line shape of mechanical strain origin would certainly vanish in the phase-coexistence region of isotherm in the absence of $\rho-\varepsilon_{\mathrm{s}}$ coupling, a nonhorizontal curved line shape would still remain in the isotherm due to the existence of intrinsic limited cooperativity of the transition. To consider how the horizontality of isotherm in the phasecoexistence region is lost upon the renormalization parameters $\alpha$ and $\beta$, we plot the actual surface pressure interval $\Delta \pi$ as a function of $\alpha$ and $\beta$ using Eq. (11) (Fig. 5). For plotting this schematic phase space, we need the values of $d \pi_{c} / d T_{c}$ and Landau coefficient ratio $B^{2} / a C$. Albrecht et al. [13] carried out to determine dipalmitolylphosphatidylcholine (DPPC) monolayer phase diagrams from the isotherm measurements. They identified a tricritical point as well as a line of first-order phase transitions on their phase diagram. From their phase diagram we take $d \pi_{c} / d T_{c}=2.35 \mathrm{mNm}^{-1} \mathrm{~K}^{-1}$ as a typical value for evaluation. The Landau coefficients of monolayers have not been reported to date. However, some efforts were made to evaluate the Landau coefficients for DPPC bilayers [34] and for liquid crystals [35,36]. Typically, the coefficients in lipid bilayers are one order of magnitude larger than those in liquid crystals. This can be simply explained by the large latent heat of gel to liquid-crystalline phase transition (we note that the latent heat of monolayers is comparative to that of lipid bilayers [13]). Most transition properties remain constant except the latent heat when the Landau coefficients are increased by the same factor [34]. In fact, we have compared the values of the Landau coefficient ratio using the coefficients reported for DPPC bilayer [34] and for nematic $\mathrm{N}$-(p-methoxybenzylidene)- $p^{\prime}$-butylaniline (MBBA) [37] and have obtained the comparative values, 2.7 (DPPC) and 4.7 (MBBA). We thus take $B^{2} / a C=3.0 \mathrm{~K}$ as a moderate value of the coefficient ratio. Using these values we have plotted $\alpha$ and $\beta$ dependences on the surface pressure interval $\Delta \pi$ in Fig. 5. The calculated values of $\Delta \pi$ are reasonable and the values previously reported for nonhorizontal isotherms lie within $\Delta \pi$ for chosen ranges of $\alpha$ and $\beta$. From this schematic phase plot we can see how the horizontality of the isotherm is lost depending upon the control parameters $\alpha$ and $\beta$. The horizontality of an isotherm is kept only on the $\beta=0$ line. Therefore, $\beta$ can be identified as the key control parameter of nonhorizontality in the isotherm. At the constant $\beta(\neq 0)$, $\Delta \pi$ is weakly dependent on $\alpha$. A negative fourth-order term in the Landau free energy creates an energy barrier in the freeenergy landscape, leading to a first-order transition. In Eq. (2) the renormalized parameters $\alpha, \beta$ are expected to decrease monotonously with increasing surface pressure since the density jump at the first-order transition decreases monotonously with increasing surface pressure upon approaching the tricritical point [13]. From the above argument, the relative contribution of $2 \mathrm{D}$ strain energy on nonhorizontality of isotherms is expected to decrease with increasing surface pressure. The size of the cooperative transforming clusters was, on the other hand, observed to increase upon approaching the tricritical point [13]. This suggests that the cooperativity of transition plays a dominant role for the nonhorizontal isotherm behavior in the coexistence region with increasing surface pressure. It may be instructive to compare our results with those by Arriaga et al. [16]. They ascribed the origin of nonhorizontal isotherm plateau in the coexistence region and the subsequent nonzero compression rigidity to kinetically limited growth of LC domains upon continuous compression. In their kinetic scenario, the growth of LC domains does not have enough time to incorporate molecules supplied from the surrounding LE phase if strained at high compression rates and the monolayer resists against compression, producing a finite compression rigidity. They proposed the "Plum-Cake" model to estimate the compression modulus as a function of the LC phase fraction in the coexistence region at a high compression rate limit. The model predicts an upper limit for the compression modulus of the coexisting monolayer expected for a relaxation process due to lipid changes between the LC domains and the surrounding LE phase at strain rates much higher than the characteristic growth rates of the LC domain. It is interesting to point out that the LC-phase fraction dependence of the renormalized composite compression modulus $K^{\prime}\left(=K_{0}\left(1+\gamma_{0}\right) /(1-v)\right)$ involved in the elasticity term of the free energy [Eq. (1)] shows qualitatively the similar behavior as that of the high-rate limit compression modulus given by the Plum-Cake model. In the equilibrium context, a composite elasticity term that includes the above compression 
modulus is naturally introduced into the free energy in our equilibrium mean-field approach. The minimization of the resultant free energy leads to a linear $\pi$ increase with the increased LC-phase fraction in the coexistence region. From the standpoint of a kinetic approach to the isotherm shape of monolayers in the coexistence region, it will be meaningful to investigate the relation between the effect of compression rates on LC domain growth kinetics and the curvature of the nonhorizontal isotherm slope.

For a single-component macroscopic system the Gibbs phase rule would require that the two-phase coexistence could be in thermodynamic equilibrium only at a single surface pressure at a fixed temperature. The phase rule in general describes the relationship among the degrees of freedom $(f)$ that can be independently varied, the number of chemical components $(c)$, the number of phases $(p)$, and the number of intensive variables $(i)$. Although controllable intensive fields are limited to temperature and pressure $(i=2)$ for traditional physicochemical systems, the phase rule must in general be changed with respect to the number of additional degrees of freedom for systems that is subjected to varying external fields such as electrical, magnetic, or mechanical ones. It must then be stated as $f=i+c-p$ in general form for any integer value $i$ depending on the experiment in question, instead of the conventional form $f=2+c-p$. The strain energy generated within the monolayer can contribute to an extra degree of freedom. It would thus not be necessary to invoke any violations of the phase rule in the LE-LC transition in the presence of a long-ranged strain field. Our strain-renormalized surface pressure $\pi_{v}^{\text {res }}[\mathrm{Eq}$. (5)] in the phase-coexistence region is indeed consistent with the phase rule modified by the strain energy. It was reported that an apparent contradiction with the Gibbs phase rule is resolved by the presence of long-ranged elastic interactions for the temperature-dependent phase coexistence between condensed (crystalline) phases where the free energies of the two phases are modified by a mechanical strain energy [23,24]. From the above considerations the diffuse first-order transition in monolayers could be caused not only by limited cooperativity of the constituent molecules within submicroscopic clusters but also by molecular density-2D strain coupling, causing two different shapes of isotherm. We should note that $\rho-\theta$ coupling alone does not lead to the appearance of a finite linear slope in the isotherm [Fig. 4(c)]. This coupling term, however, makes the surface pressure during the LE-LC transition lower effectively. In fact, the lowering of transition midpoint surface pressure can be caused if at least one of $\alpha$ and $\beta$ is nonzero [Eq. (9)].

Here let us remember that an isothermal lateral compressibility is one of the thermodynamic response functions that describe the response of the order parameter to a field conjugate to it [38]. Response functions are closely related to equilibrium fluctuations of order parameters in the system. In this respect, the appearance of a compressibility plateau $[13,19,20,25,33]$ in the LE-LC phase-coexistence region means that molecular density fluctuations remains almost constant and therefore that they do not decay easily even across at the midpoint of the transition. This indicates that $\rho-\varepsilon_{\mathrm{s}}$ coupling plays a significant role for the persistence of equilibrium molecular density fluctuations or for the delay of transition progress in the nonhorizontal linear $\pi$ increase regime of the isotherm. The $2 \mathrm{D}$ free-energy contour plots (Fig. 2) might provide us with some useful information about the persistence of equilibrium density fluctuations in the phase-coexistence region. We find that the presence of $\rho-\varepsilon_{\mathrm{s}}$ coupling leads to the formation of an asymmetric energy barrier between the LE and the LC phases, regardless of whether $\rho-\theta$ coupling is present or not [Figs 2(c) and $2(\mathrm{~d})]$. The development of such an asymmetric energy barrier due to $\rho-\varepsilon_{\mathrm{s}}$ coupling, assisted by a significant increase in the energy asymmetry $\Delta \mu$, would provide the possibility of producing a substantial driving force $\Delta_{\mathrm{LC} \rightarrow \mathrm{LE}}$ from the energetically metastable, majority LC phase to the energetically stable, minority LE phase even beyond the transition midpoint [inset in Fig. 3(b)]. This might provide a microscopic basis for the persistence of equilibrium density fluctuations manifested macroscopically as the appearance of a compressibility plateau.

\section{CONCLUSIONS}

In this paper we have presented a generalized approach based on a phenomenological Landau free energy to investigate the coupling effects of molecular area density with spontaneous collective chain tilt and 2D strain inside the LC domain on the nonhorizontal isotherm shape in the first-order LE-LC phase transition in Langmuir monolayers. This theoretical approach has the advantage that one can gain intuitive physical insights into the effects of the above mechanical degrees of freedom on the evolution of the LE-LC phase transition. The constructed Landau free energy includes surface pressure terms in the lowest-order coefficient and lateral density-chain tilt-2D strain coupling renormalization terms in the fourth-order coefficient. As thermodynamic consequences of strain renormalization in the Landau expansion, we can get some useful information on the strength of the first-order transition and on the possible existence of isotherm slope and if any, on its shape in a strained monolayer. As a main result of this phenomenological approach it is found that the existence of a finite lateral density-2D strain coupling in the transition region must be essential for the occurrence of a linear increase of surface pressure with decreasing molecular area. This result can also explain the apparent violation of the phase rule due to a contribution from the strain energy to the total degrees of freedom. In 2D contour plots calculated from our derived Landau free energy, the presence of a finite molecular density2D strain coupling might cause some significant driving force for the majority LC phase to the minority LE phase beyond the transition midpoint, leading to the possibility of the delayed LE to LC transition due to the persistence of the LE phase. From the above arguments, the appearance of a compressibility plateau as often reported before in lipid monolayers might be regarded as a natural macroscopic manifestation of the persistence of equilibrium density fluctuations due to residual LE phase caused by the molecular lateral density-2D strain coupling.

\section{APPENDIX A: EXCESS 2D STRAIN ENERGY TERM IN THE LANDAU FREE ENERGY}

Let us consider a circular LC domain embedded in a circular LE matrix (Fig. 6). Since we are concerned with the 


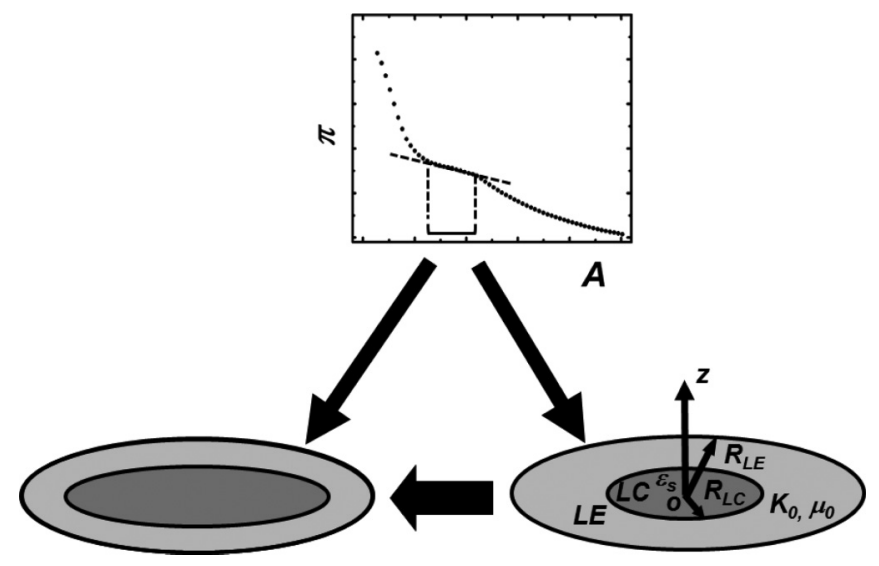

FIG. 6. Correspondence between $\pi-A$ isotherm and the growth of an LC domain surrounded by a circular LE matrix in the two-phase coexistence region of a monolayer.

nonhorizontal slope region of an isotherm appearing immediately after the onset of the two-phase coexistence in the first-order LE-LC transition, we assume that LC domains are well separated from each other. Thus we consider the region $(0 \leqslant v \leqslant 0.8)$ in which the interactions between LC domains could definitely be ignored, where $v=\left(R_{\mathrm{LC}} / R_{\mathrm{LE}}\right)^{2}$ is the area fraction of grown LC phase to the total area. We may regard $v$ as a direct counterpart of the molecular density $\left(A^{-1}\right)$ in the isotherm as described in Sec. II A. We use cylindrical coordinates for the symmetrical reason, with the origin at the center of a circular LC domain and the $z$ axis parallel to the normal to the domain. Assuming that the monolayer is uniformly compressed from all directions in the phasecoexistence region, the tangential displacements are zero and the displacement vector $\boldsymbol{u}$ is purely radial and is only a function of $r, u_{r}=u(r)$. Hence curl $\boldsymbol{u}=\mathbf{0}$ and using the equation of equilibrium, $2(1-\sigma) \operatorname{grad} \operatorname{div} \boldsymbol{u}-(1-2 \sigma)$ curl $\operatorname{curl} \boldsymbol{u}=0$ from the linear elastic theory [39], we have

$$
\nabla \cdot u=\frac{1}{r} \frac{d(r u)}{d r} \equiv 2 a=\operatorname{Tr}(\hat{\varepsilon})=\varepsilon_{\mathrm{s}}(a: \text { constant }),
$$

where $\varepsilon_{\mathrm{s}}=\operatorname{Tr}(\hat{\varepsilon})$ is a $2 \mathrm{D}$ strain inside the LC domain (2D strain is equivalent to the divergence of a displacement field). An LC domain increases in size and a misfit strain $\varepsilon_{\mathrm{s}}$ develops due to the LE-LC boundary continuity requirement with increasing surface pressure in the phase-coexistence region (Fig. 6). The radial displacement field $u_{r}$ within or outside an LC domain can be written as

$$
u_{r}^{\mathrm{LC}}=a_{1} r\left(r \leqslant R_{\mathrm{LC}}\right) \text { and } u_{r}^{\mathrm{LE}}=a_{2} r+\frac{b_{2}}{r}\left(R_{\mathrm{LC}}<r \leqslant R_{\mathrm{LE}}\right),
$$

where $R_{\mathrm{LE}}$ and $R_{\mathrm{LC}}$ are LE and LC domain radii, respectively. $a_{1}, a_{2}$, and $b_{2}$ are constants to be determined. Boundary conditions of this problem are determined from the two requirements: (i) The displacement field vanishes at the boundary, $r=R_{\mathrm{LE}}$ of parent phase considered; (ii) the strain field is continuous at the LE-LC phase boundary, $r=R_{\mathrm{LC}}$.
From the first condition,

$$
a_{2} R_{\mathrm{LE}}+\frac{b_{2}}{R_{\mathrm{LE}}}=0
$$

From the second condition,

$$
a_{1} R_{\mathrm{LC}}=a_{2} R_{\mathrm{LC}}+\frac{b_{2}}{R_{\mathrm{LC}}} .
$$

From the above equations, we obtain the following coefficients:

For LC domain

$$
a_{1}=\frac{\varepsilon_{\mathrm{s}}}{2}
$$

For LE domain

$$
\begin{aligned}
& a_{2}=-\frac{b_{2}}{R_{\mathrm{LE}}^{2}}=-\frac{\varepsilon_{s}}{2} \frac{R_{\mathrm{LE}}^{2}}{R_{\mathrm{LE}}^{2}-R_{\mathrm{LC}}^{2}}=\frac{\varepsilon_{\mathrm{s}}}{2} \frac{v}{v-1}, \\
& b_{2}=\frac{\varepsilon_{\mathrm{s}}}{2} \frac{R_{\mathrm{LC}}^{2} R_{\mathrm{LE}}^{2}}{R_{\mathrm{LE}}^{2}-R_{\mathrm{LC}}^{2}}=\frac{\varepsilon_{\mathrm{s}}}{2} \frac{R_{\mathrm{LC}}^{2}}{1-v} .
\end{aligned}
$$

We note that the strain field $u_{r}^{\mathrm{LE}}$ in the LE region is expressed by the $2 \mathrm{D}$ strain $\varepsilon_{\mathrm{s}}$ within the LC region through the boundary conditions. Since we are in the Hooke's law regime, the free energy of a deformed body $F$ is obtained from the fact that $F$ is quadratic in the strain tensor [39]:

$$
F=\frac{1}{2} \sigma_{i k} u_{i k}, \sigma_{i k}: \text { stress tensor, } u_{i k}: \text { strain tensor. }
$$

The excess free energy of the LE region $\left(R_{\mathrm{LC}}<r \leqslant R_{\mathrm{LE}}\right)$ is (per unit area of the LC domain) as follows:

$$
\therefore \Delta G_{\varepsilon_{\mathrm{s}}}^{\mathrm{LE}}=\frac{1}{2 \pi R_{\mathrm{LC}}^{2}} \int_{R_{\mathrm{LC}}}^{R_{\mathrm{LE}}}\left(\sigma_{r r} u_{r r}+\sigma_{\varphi \varphi} u_{\varphi \varphi}\right) 2 \pi r d r
$$

The stress tensor $\sigma_{i k}$ is [39]

$$
\sigma_{i k}=K_{0} u_{l l} \delta_{i k}+2 \mu_{0}\left(u_{i k}-\frac{1}{3} \delta_{i k} u_{l l}\right),
$$

where $K_{0}$ and $\mu_{0}$ are compression and shear moduli of the LE phase, respectively.

The components of the stress tensor in cylindrical coordinates are

$$
\begin{aligned}
\sigma_{r r} & =K_{0}\left(u_{r r}+u_{\varphi \varphi}\right)+2 \mu_{0}\left(u_{r r}-\frac{1}{3}\left(u_{r r}+u_{\varphi \varphi}\right)\right) \\
& =2 a_{2} K_{0}+2 \mu_{0}\left(\frac{a_{2}}{3}-\frac{b_{2}}{r^{2}}\right), \\
\sigma_{\varphi \varphi} & =K_{0}\left(u_{r r}+u_{\varphi \varphi}\right)+2 \mu_{0}\left(u_{\varphi \varphi}-\frac{1}{3}\left(u_{r r}+u_{\varphi \varphi}\right)\right) \\
& =2 a_{2} K_{0}+2 \mu_{0}\left(\frac{a_{2}}{3}+\frac{b_{2}}{r^{2}}\right) .
\end{aligned}
$$

The components of the strain tensor in cylindrical coordinates are

$$
\begin{aligned}
& u_{r r}=\frac{\partial u_{r}}{\partial r}=a_{2}-\frac{b_{2}}{r^{2}}, \\
& u_{\varphi \varphi}=\frac{\partial u_{\varphi}}{\partial \varphi}+\frac{u_{r}}{r}=a_{2}+\frac{b_{2}}{r^{2}} .
\end{aligned}
$$


Therefore,

$$
\begin{aligned}
\therefore & \sigma_{r r} u_{r r}+\sigma_{\varphi \varphi} u_{\varphi \varphi} \\
= & \left\{2 a_{2} K_{0}+2 \mu_{0}\left(\frac{a_{2}}{3}-\frac{b_{2}}{r^{2}}\right)\right\}\left(a_{2}-\frac{b_{2}}{r^{2}}\right) \\
& +\left\{2 a_{2} K_{0}+2 \mu_{0}\left(\frac{a_{2}}{3}+\frac{b_{2}}{r^{2}}\right)\right\}\left(a_{2}+\frac{b_{2}}{r^{2}}\right) \\
= & \left(4 K_{0}+\frac{4}{3} \mu_{0}\right) a_{2}{ }^{2}+\frac{4 \mu_{0} b_{2}{ }^{2}}{r^{4}} .
\end{aligned}
$$

Finally the excess free energy of surrounding LE region is expressed as follows:

$$
\begin{aligned}
\therefore & \Delta G_{\varepsilon_{\mathrm{s}}}^{\mathrm{LE}} \\
& =\frac{1}{2 \pi R_{\mathrm{LC}}{ }^{2}} \int_{R_{\mathrm{LC}}}^{R_{\mathrm{LE}}}\left\{\left(4 K_{0}+\frac{4}{3} \mu_{0}\right) a_{2}{ }^{2}+\frac{4 \mu_{0} b_{2}^{2}}{r^{4}}\right\} 2 \pi r d r \\
& =\frac{\varepsilon_{\mathrm{s}}^{2}}{2} \frac{1}{1-v}\left\{\left(K_{0}+\frac{1}{3} \mu_{0}\right) v+\mu_{0}\right\} .
\end{aligned}
$$

We see that the excess elastic energy $\Delta G_{\varepsilon_{\mathrm{s}}}^{\mathrm{LE}}$ of the LE matrix is associated with the spontaneous $2 \mathrm{D}$ strain $\varepsilon_{\mathrm{s}}$ within the grown LC domain weighted by the factor $v /(1-v)$ reflecting the growth of LC domain. Similarly, from the excess free energy of an LC domain $\left(r=R_{\mathrm{LC}}\right)$

$$
\Delta G_{\varepsilon_{\mathrm{LC}}}^{\mathrm{LC}}=\Delta S F=\frac{1}{2} \pi R_{\mathrm{LC}}^{2}\left(\sigma_{r r} u_{r r}+\sigma_{\varphi \varphi} u_{\varphi \varphi}\right),
$$

it is (per unit area of the LC domain) as follows:

$$
\begin{aligned}
\Delta G_{\varepsilon_{\mathrm{s}}}^{\mathrm{LC}} & =\frac{1}{2} \pi R_{\mathrm{LC}}{ }^{2}\left(4 a_{1}^{2} K_{0}+\frac{4}{3} \mu_{0} a_{1}^{2}\right) / \pi R_{\mathrm{LC}}{ }^{2} \\
& =\frac{1}{2} K_{0} \varepsilon_{\mathrm{s}}{ }^{2}+\frac{1}{6} \mu_{0} \varepsilon_{\mathrm{s}}{ }^{2} .
\end{aligned}
$$

Finally, the total excess elastic energy due to the development of 2D strain field as the LE to LC transition proceeds, is expressed as

$$
\begin{aligned}
\Delta G_{\varepsilon_{\mathrm{s}}} & =\Delta G_{\varepsilon_{\mathrm{s}}}^{\mathrm{LE}}+\Delta G_{\varepsilon_{\mathrm{s}}}^{\mathrm{LC}} \\
& =\frac{\varepsilon_{\mathrm{s}}^{2}}{6(1-v)}\left(3 K_{0}+4 \mu_{0}\right) \\
& =\frac{K_{0} \varepsilon_{\mathrm{s}}^{2}}{2} \frac{1+\gamma_{0}}{1-v}, \quad \gamma_{0} \equiv \frac{4}{3} \frac{\mu_{0}}{K_{0}} .
\end{aligned}
$$

This is the second term of $\Delta G_{\text {elastic }}$ in Eq. (1).

\section{APPENDIX B: DERIVATION OF THE RESCALED FREE ENERGY}

Let us consider $\Delta G_{\rho}$ in more detail below. In an isobaric experiment carried out at some elevated $\pi$, by assuming that the LE-LC phase boundary is linear in the $\pi-T$ phase diagram with a positive slope, $d \pi_{\mathrm{c}} / d T_{\mathrm{c}}>0$ (Fig. 7), the critical temperature for transition changes from $T_{0}$ to $T_{0}+d T_{\mathrm{c}} / d \pi_{\mathrm{c}} \pi$.

Thus we have

$$
\Delta G_{\rho}=\frac{1}{2} a\left\{T-\left(T_{0}+\frac{d T_{\mathrm{c}}}{d \pi_{\mathrm{c}}} \pi\right)\right\} \rho^{2}+\frac{1}{4} B \rho^{4}+\frac{1}{6} C \rho^{6} .
$$

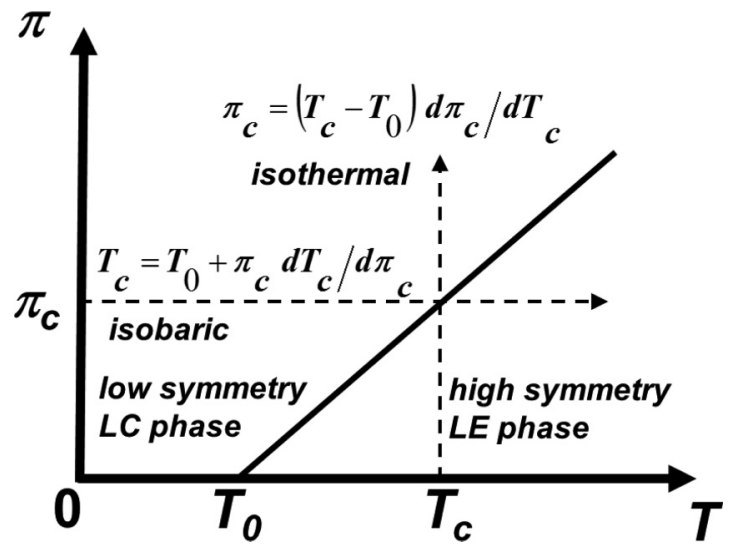

FIG. 7. Schematic illustration of the relationship between isothermal and isobaric measurements of an LE-LC phase transition with a positive slope $\partial \pi_{c} / \partial T_{c}>0$ for the phase boundary.

From Fig. 7, $\pi$ dependence of the excess free energy $\Delta G_{\rho}$ can be derived by noting that at some temperature $T$ the transition surface pressure $\pi_{c}$ can be written as $\pi_{\mathrm{c}}=$ $d \pi_{\mathrm{c}} / d T_{\mathrm{c}}\left(T-T_{0}\right)$ :

$$
\Delta G_{\rho}=-\frac{1}{2} a\left(\frac{d T_{\mathrm{c}}}{d \pi_{\mathrm{c}}}\right)\left(\pi-\pi_{\mathrm{c}}\right) \rho^{2}+\frac{1}{4} B \rho^{4}+\frac{1}{6} C \rho^{6} .
$$

Equation (B2) gives the evolution of $\rho$ with increasing $\pi$ under an isothermal condition. Replacing $\Delta G_{\rho}$ in Eq. (1) with that in Eq. (B2), we obtain an equation, providing the basis for the analysis of chain tilt-2D strain effects on the LELC transition under an isothermal condition. The interactions between the polar heads can be considered to be involved in $\pi$ and $\varepsilon_{s}$ implicitly. Since at equilibrium the monolayer must be stress free, finding the minimum of the total excess free energy in Eq. (1) with respect to $\theta_{\mathrm{LC}}$ and $\varepsilon_{s}$, respectively, we obtain $\rho$ dependence on $\theta$ and $\varepsilon_{s}$ :

$$
\begin{gathered}
\theta=-\frac{d \rho^{2}}{K_{\theta}}, \\
\varepsilon_{\mathrm{s}}=-\frac{e \rho^{2}}{K_{0}} \frac{1-v}{1+\gamma_{0}} .
\end{gathered}
$$

Thus, as $\rho$ increases, the tilt angle decreases (molecular tails orient more perpendicularly to the water surface) and the negative strain increases at constant $v$.

From Eqs. (B3) and (B4) we obtain the relation between $\varepsilon_{s}$ and $\theta$ :

$$
\varepsilon_{\mathrm{s}}=\left(\frac{K_{\theta}}{K_{0}}\right)\left(\frac{e}{d}\right) \frac{1-v}{1+\gamma_{0}} \theta .
$$

For a finite $\rho-\varepsilon_{\mathrm{S}}$ coupling $(e \neq 0)$, the spontaneous 2D negative strain grows as the tilt angle decreases at some constant $v$. Inserting Eqs. (B2)-(B4) into Eq. (1), we obtain the following equation:

$$
\begin{aligned}
\Delta G= & -\frac{1}{2} a\left(\frac{d T_{\mathrm{c}}}{d \pi_{\mathrm{c}}}\right)\left(\pi-\pi_{\mathrm{c}}\right) \rho^{2} \\
& +\frac{B}{4}\left(1-\frac{2 d^{2}}{B K_{\vartheta}}-\frac{2 e^{2}}{B K_{0}} \frac{1-v}{1+\gamma_{0}}\right) \rho^{4}+\frac{C}{6} \rho^{6} .
\end{aligned}
$$


The energy cost for the formation of LC phase should be multiplied by its area fraction $v$. The free energy $\Delta G_{v}$ for the system under the application of surface pressure is now expressed as follows:

$$
\Delta G_{\nu}=v\left(-\frac{1}{2} a\left(\frac{d T_{\mathrm{c}}}{d \pi_{\mathrm{c}}}\right)\left(\pi-\pi_{\mathrm{c}}\right) \rho^{2}+\frac{B}{4}\left(1-\frac{2 d^{2}}{B K_{\vartheta}}-\frac{2 e^{2}}{B K_{0}} \frac{1-v}{1+\gamma_{0}}\right) \rho^{4}+\frac{C}{6} \rho^{6}\right) .
$$

Rescaling the order parameter $\rho=(|B| / C)^{1 / 2} \xi$ to simplify the above expression, we finally obtain the rescaled free energy, $\Delta G_{v}^{\text {res }}[$ Eq. (2)].

[1] Organized Organic Ultrathin Films: Fundamentals and Applications, edited by K. Ariga (Wiley, Weinheim, 2012).

[2] Y. S. Lee, Self-Assembly and Nanotechnology: A Force Balance Approach (Wiley, Weinheim, 2008).

[3] Interfacial Rheology (Progress in Colloid and Interface Science), edited by R. Miller and L. Liggieri (Brill, Leiden-Boston, 2009), Vol. 1.

[4] Organized Monolayers and Assemblies: Structure, Processes and Function, edited by D. Möbius and R. Miller (Elsevier, Amsterdam, 2002).

[5] V. M. Kaganer, H. Möhwald, and P. Dutta, Rev. Mod. Phys. 71, 779 (1999).

[6] J. Lyklema, Fundamentals of Interface and Colloid Science: Liquid-Fluid Interfaces (Academic, New York, 2000).

[7] H. Möhwald, Annu. Rev. Phys. Chem. 41, 441 (1990).

[8] A. Lucero, M. R. Rodríguez Niño, A. P. Gunning, V. J. Morris, P. J. Wilde, and J. M. Rodríguez Patino, J. Phys. Chem. B 112, 7651 (2008).

[9] N. R. Pallas and B. A. Pethica, Langmuir 1, 509 (1985).

[10] Y. F. Hifeda and G. W. Rayfield, Langmuir 8, 197 (1992).

[11] A. Miller, C. A. Helm, and H. Möhwald, J. Phys. (Paris) 48, 693 (1987).

[12] H. Möhwald, in Phase Transitions in Soft Condensed Matter, edited by T. Riste and D. C. Sherrington (Plenum, New York, 1989), Vol. 145.

[13] O. Albrecht, H. Gruler, and E. Sackmann, J. Phys. (Paris) 39, 301 (1978).

[14] J. Israelachvili, Langmuir 10, 3774 (1994).

[15] E. Hatta and T. Nishimura, J. Colloid Interface Sci. 391, 111 (2013).

[16] L. R. Arriaga, I. Lopez-Montero, J. Ignes-Mullol, and F. Monroy, J. Phys. Chem. B. 114, 4509 (2010).

[17] Z. Yu, J. Jin, and Y. Cao, Langmuir 18, 4530 (2002).

[18] D. Steppich, J. Griesbauer, T. Frommelt, W. Appelt, A. Wixforth, and M. F. Schneider, Phys. Rev. E. 81, 0611231 (2010).

[19] L. K. Nielsen, T. Bjørnholm, and O. G. Mouritsen, Langmuir 23, 11684 (2007).
[20] S. Ghazvini, B. Ricke, J. A. Zasadzinski, and P. Dhar, Soft Matter 11, 3313 (2015).

[21] G. Ma and H. C. Alen, Langmuir 22, 5341 (2006).

[22] T. L. Hill, Thermodynamics for Chemists and Biologists (Addison-Wesley, Reading, MA, 1968).

[23] V. M. Kaganer, B. Jenichen, F. Schippan, W. Braun, L. Däweritz, and K. H. Ploog, Phys. Rev. Lett. 85, 341 (2000).

[24] V. M. Kaganer, B. Jenichen, F. Schippan, W. Braun, L. Däweritz, and K. H. Ploog, Phys. Rev. B 66, 045305 (2002).

[25] J. Hoyo, E. Guaus, and J. Torrent-Burgues, Eur. Phys. J. E 39, 39 (2016).

[26] J.-C. Tolédano and P. Tolédano, The Landau Theory of Phase Transitions: Application to Structural, Incommensurate, Magnetic and Liquid Crystal Systems (World Scientific, Singapore, 1987).

[27] E. R. Catapano, M. P. Lillo, C. G. Rodriguez, P. Natale, D. Langevin, F. Monroy, and I. Lopez-Montero, Langmuir 31, 3912 (2015).

[28] C. A. Helm, H. Möhwald, K. Kjaer, and J. Als-Nielsen, Biophys. J. 52, 381 (1987).

[29] B. G. Moore, C. M. Knobler, S. Akamatsu, and F. Rondelez, J. Phys. Chem. 94, 4588 (1990).

[30] O. Enders, A. Ngezahayo, M. Wiechmann, F. Leisten, and H. -A. Kolb, Biophys. J. 87, 2522 (2004).

[31] C. M. Rosetti, N. Wilke, and B. Maggio, Chem. Phys. Lett. 422, 240 (2006).

[32] K. Nag, C. Boland, N. Rich, and K. M. W. Keough, Biochim. Biophys. Acta 1068, 157 (1991).

[33] S. Ni, W. Lee, B. Li, and A. R Esker, Langmuir 22, 3672 (2006).

[34] S. Mitaku, T. Jippo, and R. Kataoka, Biophys. J. 42, 137 (1983).

[35] T. W. Stinson and J. D. Litster, Phys. Rev. Lett. 25, 503 (1970).

[36] P. Archer, I. Dierking, V. Görtz, and J. W. Goodby, Eur. Phys. J. E 25, 385 (2008).

[37] G. Vertogen and W. H. de Jeu, Thermotropic Liquid Crystals, Fundamentals (Springer, Berlin, 1988).

[38] L. E. Reichl, A Modern Course in Statistical Physics, 2nd ed. (John Wiley and Sons, New York, 1998).

[39] L. D. Landau and E. M. Lifshitz, Theory of Elasticity (Pergamon, London, 1959). 